\title{
Density Functional Theory Calculation on Electronic Structure and Optical Properties of Copper Doped $\mathrm{SnO}_{2}$
}

\author{
Weigen $\mathrm{Wu}^{\mathrm{a},{ }^{*}}$ \\ College of Electrical \& Information Engineering, Panzhihua University, Panzhihua 617000, China \\ a13980349555@139.com
}

Keywords: Band Structure; the Density of State; Reflectivity Spectrum; Absorption Spectrum.

\begin{abstract}
Based on Density Functional Theory, we investigated the optical structures and the electronic properties of $\mathrm{Cu}$ doped $\mathrm{SnO}_{2}$ with density of $4.35 \%$, including band structure, the density of state (dos), reflectivity spectrum and optical absorption spectrum. The results show that Fermi level access conduction band gradually with the doped density. It has enhanced the electrical and metal property of material. The peaks of reflectivity spectrum and absorption spectrum are correspond density of state.
\end{abstract}

\section{Introduction}

Tin dioxide is a wide gap n-type semiconductor $(\mathrm{Eg}=3.6 \mathrm{eV})$ having rutile crystal structure [1, 2]. It is used in a wide variety of technologies, including solar cells, catalysis and gas sensors [3-7]. Its' properties depend on oxidation and reduction reactions at the surface and its surface structure [8,9]. Many experimental studies have indicated that the sensing performances of tin dioxide can be enhanced by addition of different metal species [10-13]. Moreover, the influence of dopants such as $\mathrm{Cu}$, in, or $\mathrm{F}$ has been theoretically studied in the bulk of $\mathrm{SnO} 2$ in order to improve his electrical or sensing properties [14-16].

Although doped $\mathrm{Cu}: \mathrm{SnO}_{2}$ have been researched experimentally and theoretically, it is rarely discussed about the effect of electronic structures to the optical properties. So we investigated the effects of $\mathrm{Cu}$ doped $\mathrm{SnO}_{2}$ with density of $4.35 \%$. And we demonstrated the band structure, density of state (dos), reflectivity and optical absorption spectrum.

\section{Theoretical Model and Calculation Method}

Ideal $\mathrm{SnO}_{2}$ crystal is rutile structure, and belongs to P42/MNM(D4H-14) space group, and crystal constant a equal $\mathrm{b}$ are $47.4 \mathrm{~nm}$, and $\mathrm{c}$ is $31.9 \mathrm{~nm}$. The calculation model adopted in this paper are based on the model of $\mathrm{SnO}_{2}$ supercell(Fig.1). The analysis of electron structure is mainly the calculation of the energy band structure , the density of state(dos) reflectivity and absorption spectrum, which are accomplished by density functional theory.

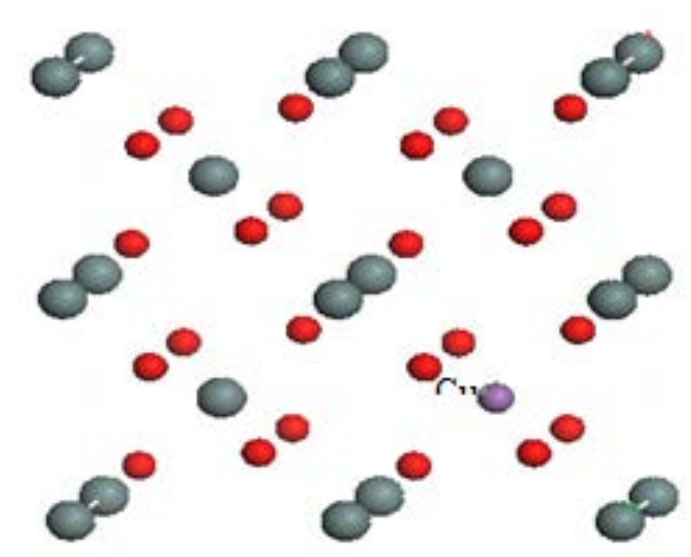

Fig.1 Supercell of Cu-doped $\mathrm{SnO}_{2}$ 


\section{Band structure}

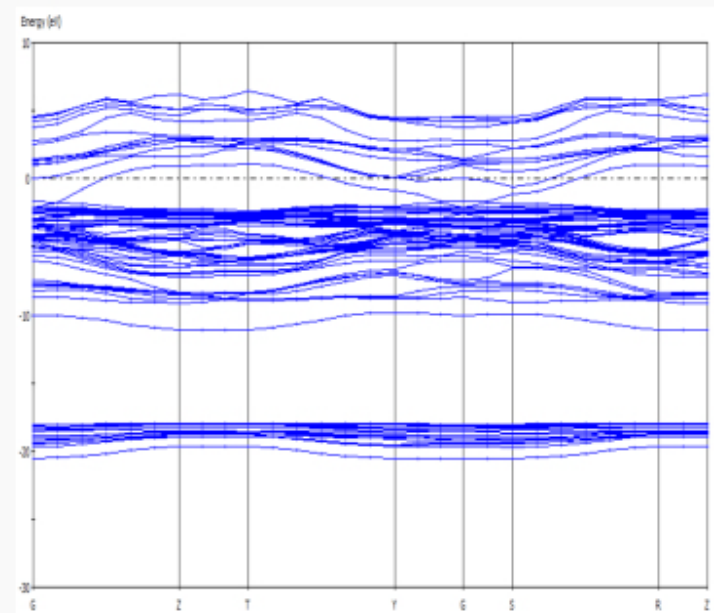

Fig.2 band structure of $\mathrm{Cu}$-doped $\mathrm{SnO}_{2}$

From Fig.2 we can see that the doped structure belongs to wind forbidden band direct band semiconductors. The top of valence band and the bottom of conduction band locate $\mathrm{G}$ from brillouin zone. Taking fermi energy level as a refence point of zero energy, we just consider near fermi level of conduction band and valence band. From the band structure we found that the numbers of energy in all band structure are markedly increased than undoped $\mathrm{SnO}_{2}[17]$, which had been contributed from $\mathrm{Cu} 5 \mathrm{~d}$ and 5s.The fermi energy level moves into conduction band. The reason is mainly that doping concentration causes free carriers have changed the energy band of $\mathrm{SnO}_{2}$ in two aspects. In one hand, high concentration carriers have brought that the fermi energy level enters the conduction band. In the other hand, the interactions among carriers generate the multi-body effect,or the overlapping between impurity band and defect band makes the gap narrow. Moreover, the effect of the former is smaller than one of the latter, which is known by calculating. We can also see that the energy band in fermi energy level had been refined, the interaction between the electrons have been reinforced. In a word, the conductivity of the material has been increased.

\section{The density of state (dos)}

Fig.3 is the density of state of $\mathrm{Cu}$-doped $\mathrm{SnO}_{2}$. Compared density of states before and after doping , we can see the marked effects of doping. And we can know that the the density of state mainly can be divided into three parts, the density of state between $-20.0 \sim-18.1 \mathrm{ev}$ is mainly contributed from O2s, $\mathrm{Cu} 5 p$, Cu5s, Sn5p and Sn5s states. The density of state between $-12.2 \sim-1.14 \mathrm{ev}$ is mainly contributed from Cu5p, Cu5s, Sn5p and Sn5s states, O2s has little contribution. the density of state between $-1.14 \sim 0 \mathrm{ev}$ is mainly contributed from Cu5p state. Nearby $0 \mathrm{ev}$, the fermi energy level moves into conduction band, doping $\mathrm{Cu}$ material has semimetal property. And an electron can obviously jump from Sn5s to O2p state, which can cause that the gravitation center of local state density on oxide position shifts toward low energy level.

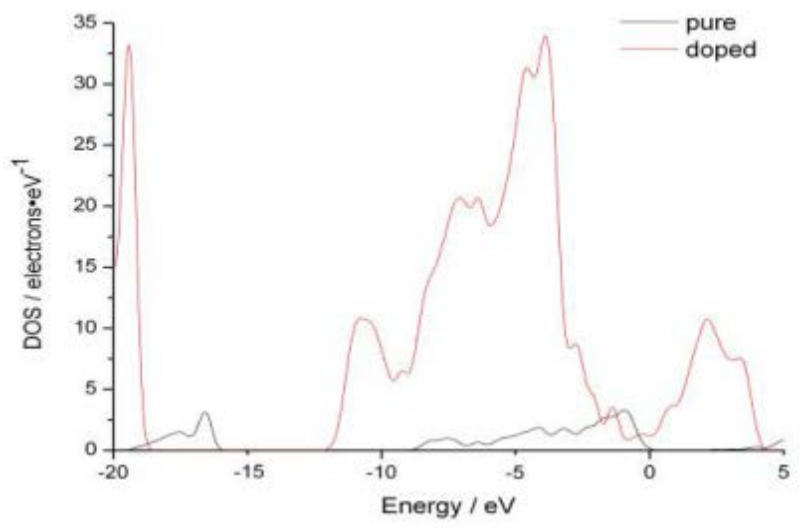

Fig.3 The density of state of $\mathrm{Cu}$-doped $\mathrm{SnO}_{2}$ 


\section{Reflectivity spectrum}

Reflectivity spectrum is a macro reflection, and the electrons in the solid material jump between energy level under the photoelectric field perturbation. From Fig.4 we can see that there are four maxima value, which locat in $1.78 \mathrm{eV}, 4.04 \mathrm{ev}, 7.29 \mathrm{ev}$ and $13.79 \mathrm{ev}$, corresponding intensity $0.08,0.11$, 0.22 and 0.39 arb.units. It is corresponding to the density of state. The intensity reaches maximum value when Frequency is $0.3913 .79 \mathrm{eV}$. The jump between energy level occurs mostly 7.29 14.0ev.

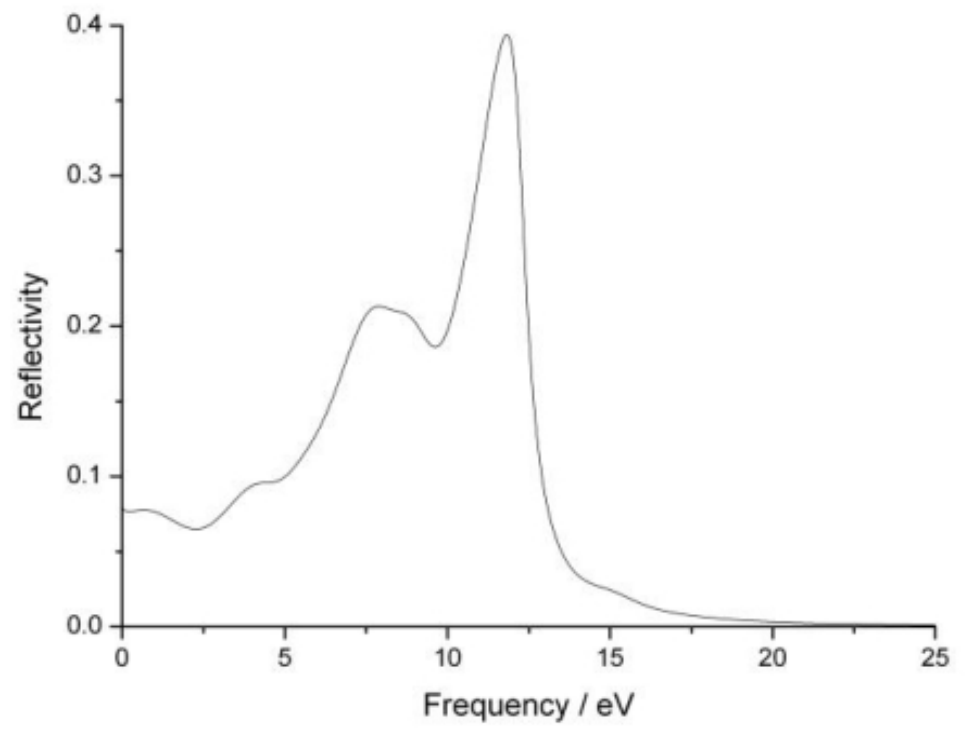

Fig.4 The reflectivity spectrum of Cu-doped $\mathrm{SnO}_{2}$

\section{Absorption spectrum}

Absorption spectrum can be expressed by function [18].

$$
I(\omega)=\sqrt{2} \omega\left[\sqrt{\varepsilon_{1}^{2}(\omega)-\varepsilon_{2}^{2}(\omega)}-\varepsilon_{1}(\omega)\right]^{\frac{1}{2}}
$$

From Fig.5 we can see that there are three summits which locate in7.81ev, 10.52ev and 14.49ev, corresponding respectively intensity $138553 \mathrm{~cm}^{-1}, 135239 \mathrm{~cm}^{-1}$ and $9484 \mathrm{~cm}^{-1}$, which is corresponding to the density of state and reflectivity spectrum. Absorption spectrum has very strongly selectivity, and mainly assimilates the light between $7.2 \mathrm{eV}$ and $12.8 \mathrm{eV}$.

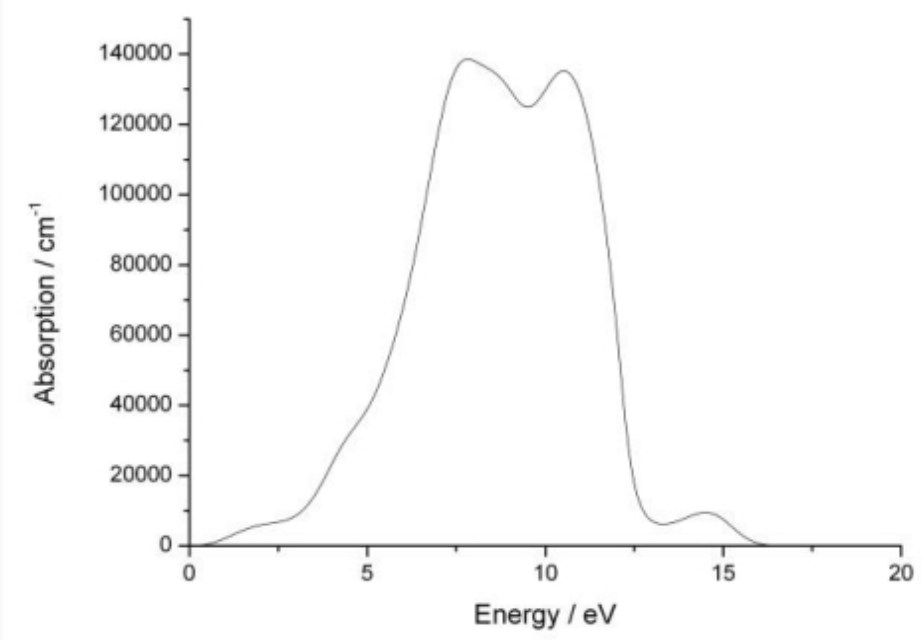

Fig.5 The absorption spectrum of Cu-doped $\mathrm{SnO}_{2}$

\section{Conclusion}

The internal relation of electron structure with conduction capability in $\mathrm{SnO}_{2}$ : $\mathrm{Cu}$ is researched by the Density Functional Theory. The simulation results are follows: 
$\mathrm{SnO}_{2}$ is theoretically previewed as a kind of direct-gap semiconductor, whose both of the bottom of conduction band and top of valence band lie to the center point $\mathrm{G}$ in Brillouin zone.

Fermi level access conduction band gradually with the doped density. It has enhanced the metal and electrical property of material.

The peaks of reflectivity Spectrum and absorption spectrum are corresponded to the density of state.

\section{References}

[1] V. E. Henrich, P. A. Cox, The Surface Science of Metal Oxides, Cambridge University Press, Cambridge, 1966.

[2] F. J. Arlinghaus, Journal of Physics and Chemistry of Solids, 35 (1974) 931.

[3] G. Korotcenkov, V. Brynzari, S. Dmitriev, Materials Science and Engineering B, 56 (1999) pp.195-204.

[4] B.W. Licznercki, et al., Sensors and Actuators B 103 (2004), p.69-75.

[5] G. Jimenez Cadena, J. Riu, F.X. Rius, Gas Sensors Based on Nanostructured Materials Analyst, 132 (2007), p.1083-1099.

[6] M. Batzill, U. Diedold, Progress in Surface Science, 79 (2005), p.47.

[7] Y. Lee, K. Lee, D. Lee, Y. Jeong, H. S. Lee, Y. Choa, Current Applied Physics, 9 (2009), p.579.

[8] D. F. Cox, T. B. Fryberger, S. Semancik, Physical Review B, 38 (1988), p.2072.

[9] I. Manassidis, J. Goniakowski, L. N. Kantorovich, M. J. Gillan, Surface Science, 339 (3) (1995), p.258-271.

[10] E. Elangovan, K. K. Ramesh, K. Ramamurthi, Solid State Communications, 130 (2004), p.523-527.

[11] G. Korotcenkov, et al., Thin Solid Films 467 (2004), p.209-214.

[12] Guoe Cheng, et al., Journal of Crystal Growth 309 (2007), p.53-59.

[13] J. Montero, J .C. Herrero, C. Guillén, Solar Energy Materials \& Solar Cells 94 (2010), p.612-616.

[14] K. C. Mishra, K. H. P. C. Johnson, Schmidt Physical Review B, 51(20) (1995) 13972.

[15] Guoqiang Qin, et al., Thin Solid Films, 517 (2009), p.3345-3349.

[16] Jian Xu, Shuiping Huang, Zhanshan Wang, Solid State Communications, 149 (2009), p.527-531.

[17] Yaming Liu, Henan Institute of Science and Technology Journal, (2011), p.78-81.

[18] Xuechu Shen, Spectroscopy and Optical Properties of Semiconductor, Science Publishing Company (2002). 